\title{
An analytical approximation of post-shock conditions in type II supernova shells
}

\author{
D. K. Nadyozhin ${ }^{1,2}$ and A. Yu. Deputovich ${ }^{1}$ \\ 1 Institute of Theoretical and Experimental Physics, B. Cheremushkinskaya St. 25, 117259 Moscow, Russia \\ 2 Max-Planck-Institut für Astrophysik, Garching, Germany
}

Received 16 October 2001 / Accepted 21 December 2001

\begin{abstract}
We describe simple analytical formulae constructed to approximate the temporal behavior of the temperature, density, and radius of various presupernova shells immediately after the arrival of the supernova shock wave. The formulae are derived from detailed hydrodynamical simulation of the supernova envelope expulsion. When used within traditional nuclear kinetic codes, the formulae are expected to be a useful tool for adjusting the resulting nuclear yields to current supernova models.
\end{abstract}

Key words. stars: supernovae: general - shock waves - nuclear reactions, nucleosynthesis, abundances

\section{Introduction}

Recent progress in observations and physics relevant to stellar nucleosynthesis (see Wallerstein et al. 1997 for a comprehensive review) has rekindled interest in the modelling of nuclear processes in stars and supernovae. It would be useful to have simple analytical approximations describing the physical conditions in the supernova shells heated and accelerated by the shock wave (SW). The purpose of the present paper is to show that this can be achieved, at least for supernovae of type II (and of types Ib, Ic as well). In these supernovae, the hydrodynamics of the envelope expulsion is virtually insensitive to the details of the supernova mechanism generating a powerful SW at the interface between the collapsing iron core and the outer envelope finally to be expelled. The only important parameter connecting the explosion mechanism and the envelope outburst is the total explosion energy $E$. This is opposite to type Ia supernovae, where one cannot disentangle the explosive thermonuclear burning from the hydrodynamics of the expulsion in which ultimately all the star turns out to be drawn.

In Sect. 1 we describe briefly the hydrodynamic simulation of the type II supernova explosions and in Sect. 2 we derive analytical approximations for the temporal behavior of the temperature, density and radius of shocked matter in different shells of $15 M_{\odot}$ and $30 M_{\odot}$ supernova models. In particular, we show that a simple exponential approximation used widely for the post-shock temperature: $T \sim \exp [-t /(3 \tau)]$ where $\tau$ is the "hydrodynamic"

Send offprint requests to: D. K. Nadyozhin, e-mail: nadezhin@vxitep.itep.ru time scale connected with the local pre-shock density $\rho$ by the expression

$\tau=\frac{446}{\sqrt{\rho}} \mathrm{s}$

is actually far from being satisfactory.

The preliminary results of this study were reported at the 10th Ringberg Castle Workshop on Nuclear Astrophysics (Nadyozhin et al. 2000).

\section{Hydrodynamic simulation}

In order to obtain an approximation of supernova SW properties useful for exploring nucleosynthesis, we have carried out a series of hydrodynamic simulations of supernovae. The calculations were done using the hydrodynamic supernova code SNV developed previously at ITEP (see, e.g., Litvinova \& Nadyozhin 1983). The code was run in its simplest mode. An explicit difference scheme was used for adiabatic hydrodynamics with the artificial viscosity being utilized to control the propagation of shock waves. The equation of state EPEOS from Blinnkov et al. (1996) was used which takes into account the black-body radiation and full Fermi-Dirac statistics for the electronpositron component. This last point turns out to be of importance for the SW properties in the SiS- and CO-shells where the number of the electron-positron pairs can become comparable with the number of "atomic" electrons (Deputovich \& Nadyozhin 1999).

Two Woosley-Weaver (1995) presupernova models of $15 M_{\odot}$ and $30 M_{\odot}$ were taken as initial conditions. The two models have red supergiant structures of radii $490 R_{\odot}$ 
Table 1. The shell structure of a $15 M_{\odot}$ presupernova.

\begin{tabular}{lcccc}
\hline \hline Shell & $\begin{array}{c}m \\
\left(M_{\odot}\right)\end{array}$ & $\begin{array}{c}R_{0} \\
\left(10^{8} \mathrm{~cm}\right)\end{array}$ & $\begin{array}{c}\rho_{0} \\
\left(\mathrm{~g} \mathrm{~cm}^{-3}\right)\end{array}$ & $\begin{array}{c}T_{0} \\
(\mathrm{~K})\end{array}$ \\
\hline SiS-bottom & 1.43 & 1.51 & $2.18 \times 10^{7}$ & $4.73 \times 10^{9}$ \\
\hline SiS-middle & 1.53 & 1.90 & $1.03 \times 10^{7}$ & $3.84 \times 10^{9}$ \\
\hline $\begin{array}{l}\text { SiS-top } \\
\text { CO-bottom }\end{array}$ & 1.63 & 2.31 & $5.28 \times 10^{6}$ & $3.33 \times 10^{9}$ \\
\hline CO-middle & 2.19 & 8.72 & $1.29 \times 10^{5}$ & $1.12 \times 10^{9}$ \\
\hline $\begin{array}{l}\text { CO-top } \\
\text { He-bottom }\end{array}$ & 2.75 & 34.3 & $1.05 \times 10^{3}$ & $2.82 \times 10^{8}$ \\
\hline He-middle & 3.60 & 161 & $3.17 \times 10^{1}$ & $7.74 \times 10^{7}$ \\
\hline $\begin{array}{l}\text { He-top } \\
\text { HHe-bottom }\end{array}$ & 4.45 & 550 & $2.12 \times 10^{-1}$ & $1.46 \times 10^{7}$ \\
\hline
\end{tabular}

Table 2. The shell structure of a $30 M_{\odot}$ presupernova.

\begin{tabular}{lcccc}
\hline \hline Shell & $\begin{array}{c}m \\
\left(M_{\odot}\right)\end{array}$ & $\begin{array}{c}R_{0} \\
\left(10^{8} \mathrm{~cm}\right)\end{array}$ & $\begin{array}{c}\rho_{0} \\
\left(\mathrm{~g} \mathrm{~cm}^{-3}\right)\end{array}$ & $\begin{array}{c}T_{0} \\
(\mathrm{~K})\end{array}$ \\
\hline SiS-bottom & 1.88 & 2.47 & $9.43 \times 10^{6}$ & $3.96 \times 10^{9}$ \\
\hline SiS-middle & 2.11 & 3.17 & $4.48 \times 10^{6}$ & $3.28 \times 10^{9}$ \\
\hline $\begin{array}{l}\text { SiS-top } \\
\text { O-bottom }\end{array}$ & 2.32 & 3.97 & $2.41 \times 10^{6}$ & $2.71 \times 10^{9}$ \\
\hline O-middle & 5.77 & 28.1 & $2.69 \times 10^{4}$ & $8.20 \times 10^{8}$ \\
\hline $\begin{array}{l}\text { O-top } \\
\text { He-bottom }\end{array}$ & 9.20 & 67.7 & $7.46 \times 10^{2}$ & $3.07 \times 10^{8}$ \\
\hline He-middle & 10.5 & 140 & $1.02 \times 10^{2}$ & $1.51 \times 10^{8}$ \\
\hline $\begin{array}{l}\text { He-top } \\
\text { HHe-bottom }\end{array}$ & 11.8 & 547 & $7.72 \times 10^{-2}$ & $1.82 \times 10^{7}$ \\
\hline
\end{tabular}

and $1025 R_{\odot}$, and iron cores of $1.4 M_{\odot}$ and $1.85 M_{\odot}$, respectively. They have a rather complicated chemical structure. For our purpose, it seems to be adequate to divide each model into three shells of special importance for nucleosynthesis: silicon-sulfur ( $\mathrm{SiS})$, carbon-oxygen $(\mathrm{CO}$, for $\left.15 M_{\odot}\right)$, or oxygen $\left(\mathrm{O}\right.$, for $\left.30 M_{\odot}\right)$, and helium $(\mathrm{He})$. Tables 1 and 2 summarize the properties of the onion-like shell structure of these models.

The supernova outburst was simulated through the initial conditions by instantaneous liberation of the explosion energy $E$ in a narrow mass layer $\left(\delta m \approx 0.1 M_{\odot}\right)$ located just at the outer boundary of the iron core, below the SiS-shell. The bottom of this layer was considered in the internal boundary condition as a rigid wall at rest where the velocity remained equal to zero at all times. Several modes of the energy release (with different ratios of the thermal to kinetic energy component) have been tested in the preliminary calculations to ensure that our results are independent of the specific way of energy release.

\section{Analytical approximations}

The main parameters determining the temporal behavior of shocked matter are the energy of the explosion $E$ which comes from the supernova mechanism, unfortunately not yet well known, the identity of the shell under consideration $(\mathrm{SiS}, \mathrm{CO}, \mathrm{He})$, the initial (pre-shock) density $\rho_{0}$ and the radius $R_{0}$ of the shell. The initial temperature $T_{0}$ of the shell is virtually unimportant since the $\mathrm{SW}$ is always very strong.

In order to construct the approximation formulae, we have to identify such combinations of these parameters which can serve as useful units to measure the post-shock temperature $T_{\mathrm{p}}$ and time $t$. The best candidate for the temperature unit is the Weaver-Woosley (1980) estimate for the SW peak temperature. This temperature, that we designate as $T_{\mathrm{WW}}$, is equal to the temperature of the black-body radiation which uniformly fills the sphere of the $\mathrm{SW}$ radius $R_{0}$ and whose total energy is equal to the supernova explosion energy $E: \frac{4 \pi}{3} R_{0}^{3} a T_{\mathrm{WW}}^{4}=E$. Thus, $T_{\mathrm{WW}}$ scales as $E^{0.25} R_{0}^{-0.75}$ and reproduces the SW peak temperature $T_{\mathrm{p}}$ with a good accuracy (within $\pm 20 \%$ ) for all the SW way through the presupernova shell structure of interest for nucleosynthesis (Deputovich \& Nadyozhin 1999). Such an amazing accuracy turns out to be connected with a coincidental compensation between the contribution of the kinetic energy to the total explosion energy $E$ (just behind a strong SW, the kinetic energy is equal to the thermal one) and the excess of $T_{\mathrm{p}}$ over the mean temperature of shocked matter (Deputovich \& Nadyozhin 1999). The temperature $T_{\mathrm{WW}}$ fails, however, to follow $T_{\mathrm{p}}$ at the outermost layers during the SW breakout through the presupernova photosphere.

An appropriate estimate of the characteristic time $t_{\mathrm{u}}$ can be made from the following consideration. The time scale of the post-shock expansion is controlled by the speed of sound, $v_{\text {sound }}$, in shocked matter and is equal to $t_{\mathrm{u}}=R_{0} / v_{\text {sound }}$ by the order of magnitude. Taking into account that $v_{\text {sound }}^{2}=\gamma P / \rho$, one finds $v_{\text {sound }} \sim 1 / \sqrt{\rho}$ for the radiation dominated media where $\gamma=4 / 3, P=a T^{4} / 3$. We see that the dependence of the characteristic time $t_{\mathrm{u}}$ on density turns out to be just opposite to that of $\tau$ from Eq. (1). This correct way to estimate the time scale of the post-shock expansion was used earlier by Thielemann et al. (1979). The final expressions for $T_{\mathrm{WW}}$ and $t_{\mathrm{u}}$ are

$T_{\mathrm{WW}}=\left(\frac{3 E}{4 \pi a R_{0}^{3}}\right)^{1 / 4}=2.37 \times 10^{9} E_{51}^{0.25}\left(R_{09}\right)^{-0.75} \mathrm{~K}$

$t_{\mathrm{u}}=3.83 \times 10^{-3} \rho_{0}^{0.5} E_{51}^{-0.5}\left(R_{09}\right)^{2.5} \mathrm{~s}$,

where $E_{51} \equiv E / 10^{51} \mathrm{erg}$ and $R_{09} \equiv R_{0} / 10^{9} \mathrm{~cm}$. The above expression for $t_{\mathrm{u}}=R_{0} / v_{\text {sound }}$ was obtained by using $P=a T_{\mathrm{WW}}^{4} / 3, \gamma=4 / 3$, and $\rho=7 \rho_{0}$ for the post-shock pressure, adiabatic index, and density.

The natural units of length and density are the initial radius $R_{0}$ and pre-shock density $\rho_{0}$ of the layer crossed by the SW. 
Table 3. The structural coefficients for a $15 M_{\odot}$ supernova.

\begin{tabular}{lllll}
\hline \hline Shell & $\xi_{\mathrm{p}}$ & $\xi_{\mathrm{T}},\left(\xi_{\mathrm{T} 1}\right)$ & $\xi_{\mathrm{r}},\left(\xi_{\mathrm{r} 1}, \xi_{\mathrm{r} 2}\right)$ & $\xi_{\mathrm{SW}}$ \\
\hline SiS-middle & 1.1 & $2.2,(13)$ & $1.1,(0.5,2.2)$ & 0.54 \\
SiS-top/CO-bottom & 1.1 & $2.2,(13)$ & $1.1,(0.5,2.2)$ & 0.74 \\
CO-middle & 0.95 & 1.4 & 1.25 & 1.10 \\
CO-top/He-bottom & 0.85 & 0.90 & 0.85 & 0.98 \\
He-middle & 0.90 & 1.5 & 1.3 & 0.69 \\
He-top/HHe-bottom & 0.75 & 1.3 & 1.1 & 0.54 \\
\hline
\end{tabular}

In terms of the above units, the results of our hydrodynamic calculations for different supernova layers of a $15 M_{\odot}$ and a $30 M_{\odot}$ model can be approximated by the following simple relations for the temporal evolution of temperature, density, and radius of a Lagrangian layer:

$T(t)=\frac{T_{\mathrm{p}}}{1+\xi_{\mathrm{T}} t / t_{\mathrm{u}}}, \quad T_{\mathrm{p}}=\xi_{\mathrm{p}} T_{\mathrm{WW}}$,

$\rho(t)=\rho_{\mathrm{p}}\left(\frac{T}{T_{\mathrm{p}}}\right)^{3}, \quad \rho_{\mathrm{p}}=7 \rho_{0}$,

$R(t)=R_{0}\left(1+\xi_{\mathrm{r}} t / t_{\mathrm{u}}\right)$,

where $T_{\mathrm{p}}$ and $\rho_{\mathrm{p}}$ are the peak temperature and density of shocked matter while $\xi_{\mathrm{p}}, \xi_{\mathrm{T}}$, and $\xi_{\mathrm{r}}$ are the dimensionless structural coefficients chosen to fit the hydrodynamic calculations as close as possible. Equations (4)-(6) imply that the SW reaches the layer at $t=0$.

In the case of neutrino nucleosynthesis (see, e.g., Nadyozhin et al. 1998), one has to take into account that the neutrino flux scales as $L_{\nu}\left(t+\delta t_{\mathrm{SW}}+\Delta t_{\mathrm{SW}}\right) / R^{2}(t)$ where $L_{\nu}$ is the partial ${ }^{1}$ neutrino luminosity, $\Delta t_{\mathrm{SW}}$ is the total time that the SW takes to reach the layer under consideration, whereas $\delta t_{\mathrm{SW}}$ is the time interval between the beginning of the neutrino burst and the moment of the SW transition from a steady accreting state into an outgoing blast wave; $\delta t_{\mathrm{SW}}$ depends on the supernova mechanism (typically $\delta t_{\mathrm{SW}} \approx 0.1 \mathrm{~s}$ ).

The values of $\Delta t_{\mathrm{SW}}$, obtained in our hydrodynamical calculations, can be approximated with an accuracy of $\approx 20 \%$ by the following formula:

$\Delta t_{\mathrm{SW}}=\xi_{\mathrm{SW}} E_{51}^{-0.38}\left(R_{0} / 10^{9} \mathrm{~cm}\right)^{1.4} \mathrm{~s}$,

where $\xi_{\mathrm{SW}}$ is another dimensionless fitting coefficient. In order to approximate $\Delta t_{\mathrm{SW}}$ by the simplest way for all the three presupernova shells under consideration, we had to choose the irrational powers in Eq. (7). Note that Eq. (7) is close to the Sedov solution for the SW propagating in a power law media with $\rho \sim 1 / r^{2}$. In that case, the Sedov solution gives $\Delta t_{\mathrm{SW}} \sim E^{-0.5} R_{0}^{1.5}$.

The numerical values of the above fitting coefficients are presented in Tables 3 and 4 for different layers of $15 M_{\odot}$ and $30 M_{\odot}$ supernovae.

We did not present the approximations for the bottom of the SiS-shell. The reason is that this layer is very close to

\footnotetext{
${ }^{1}$ Related to those types of neutrino which actually take part in nucleosynthesis.
}

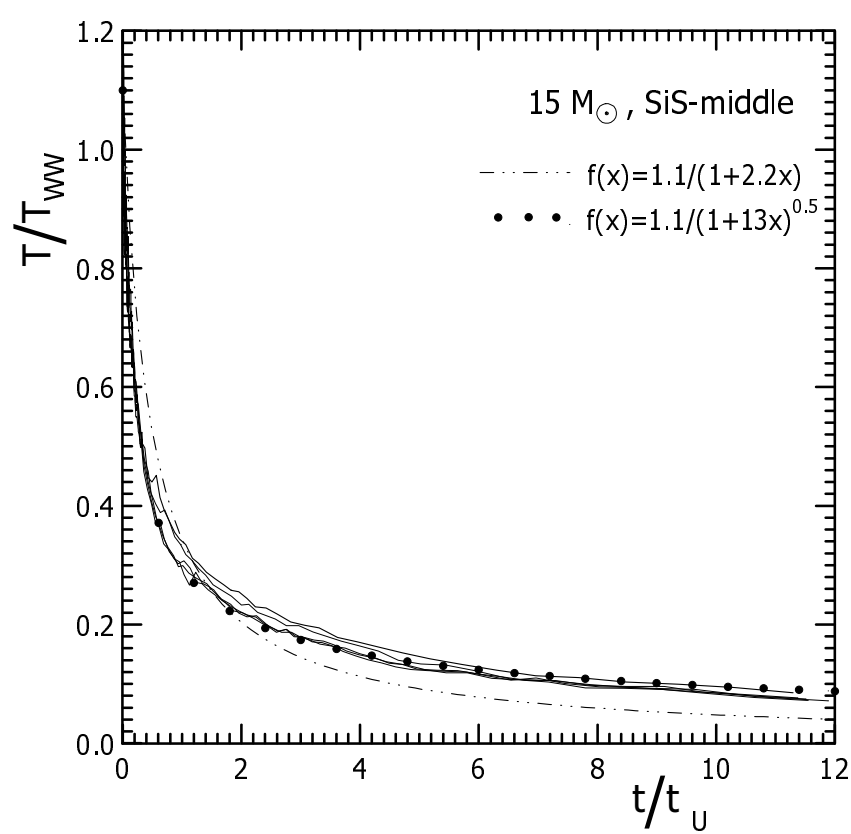

Fig. 1. The temperature in the middle of the SiS-shell versus time for a $15 M_{\odot}$ model. The full wavy lines stand for the hydrodynamic calculations while a dash-dot line and black dots show the analytical approximations.

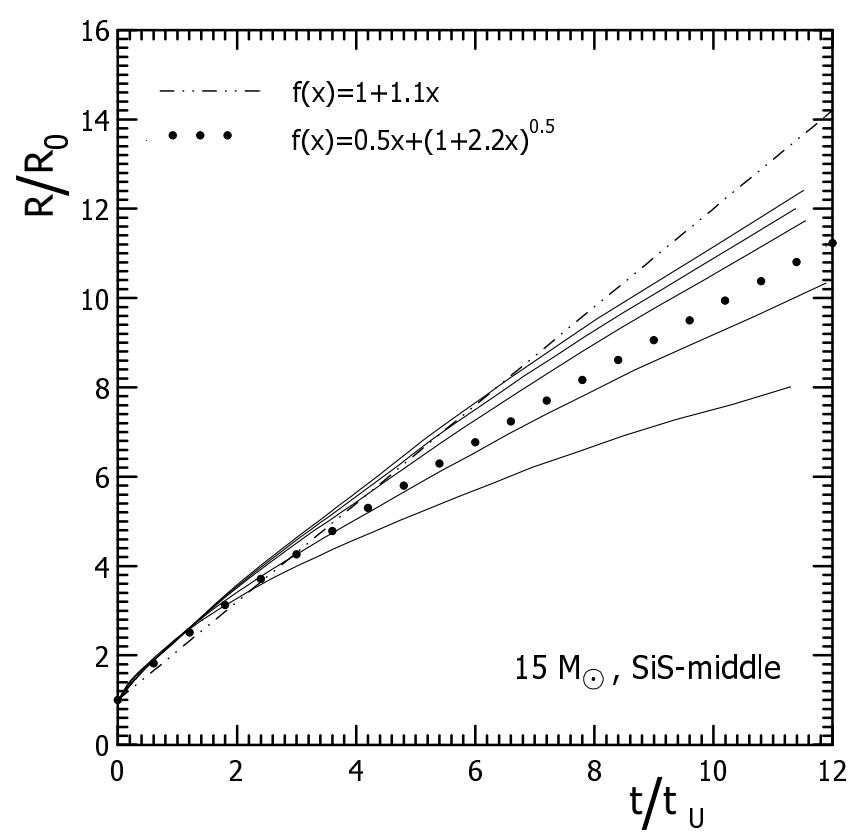

Fig. 2. Same as Fig. 1, but for the radius.

Table 4. The structural coefficients for a $30 M_{\odot}$ supernova.

\begin{tabular}{lllll}
\hline \hline Shell & $\xi_{\mathrm{p}}$ & $\xi_{\mathrm{T}},\left(\xi_{\mathrm{T} 1}\right)$ & $\xi_{\mathrm{r}},\left(\xi_{\mathrm{r} 1}, \xi_{\mathrm{r} 2}\right)$ & $\xi_{\mathrm{SW}}$ \\
\hline SiS-middle & 1.07 & $1.4,(8.0)$ & $1.0,(0,4.5)$ & 0.36 \\
SiS-top/O-bottom & 1.0 & $1.3,(6.0)$ & $1.0(0,4.5)$ & 0.49 \\
O-middle & 0.80 & 2.0 & 1.1 & 1.52 \\
O-top/He-bottom & 0.80 & 1.1 & 0.90 & 1.40 \\
He-middle & 0.80 & 1.3 & 1.1 & 1.18 \\
He-top/HHe-bottom & 0.80 & 1.0 & 1.0 & 0.58 \\
\hline
\end{tabular}




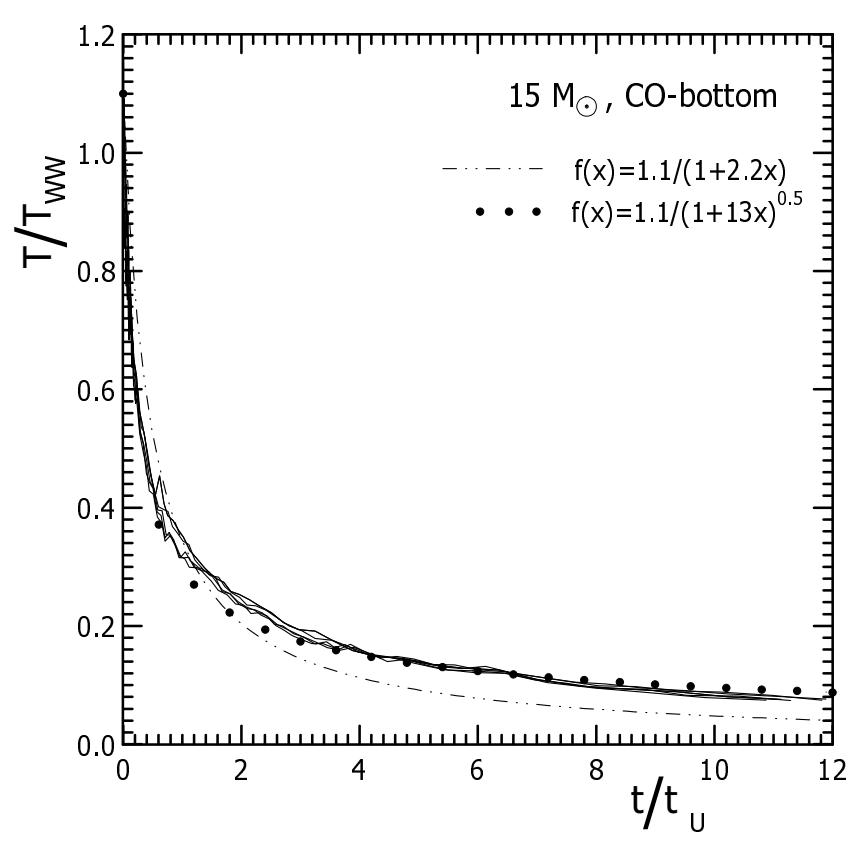

Fig. 3. Same as Fig. 1, but for the bottom of the CO-shell.

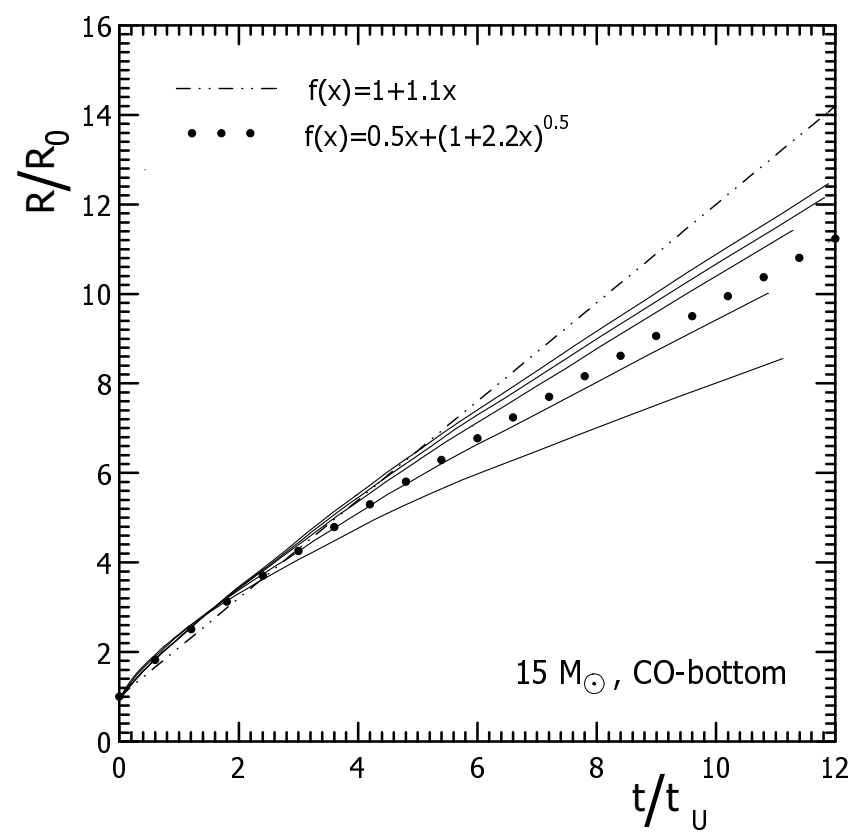

Fig. 4. Same as Fig. 2, but for the bottom of the CO-shell.

the place of explosive energy release and the hydrodynamical flow is still influenced by the details of the artificial initial conditions used in our calculations. Moreover, in the SiS-shell our adiabatic hydrodynamics might be violated owing to the energy release in the SW-induced thermonuclear reactions. Thus, our results for the SiS-shell should be considered as being preliminary.

Figures 1-22 show the temporal evolution of the postshock temperature and radius for both the models $\left(15 M_{\odot}\right.$ and $\left.30 M_{\odot}\right)$. The results of our hydrodynamic calculations are shown in each figure by a bunch of full lines related to a wide range of the supernova explosion energies covered in our calculations: $1 \times 10^{51} \lesssim E \lesssim 2 \times 10^{52} \mathrm{erg}$. The dash-dot

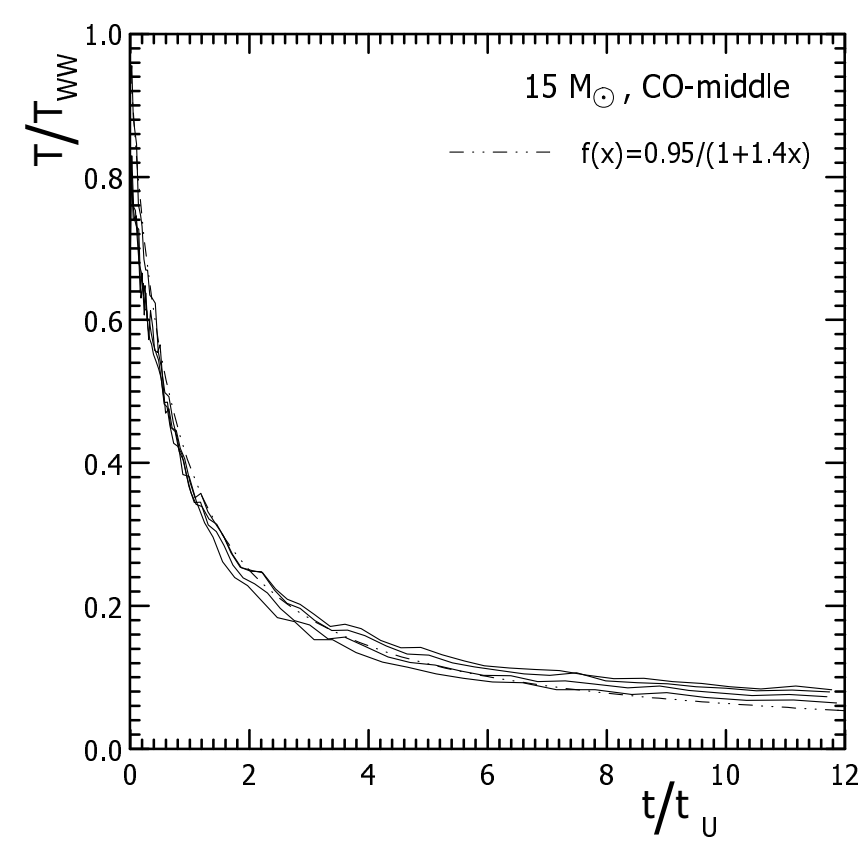

Fig. 5. Same as Fig. 1, but for the middle of the CO-shell.

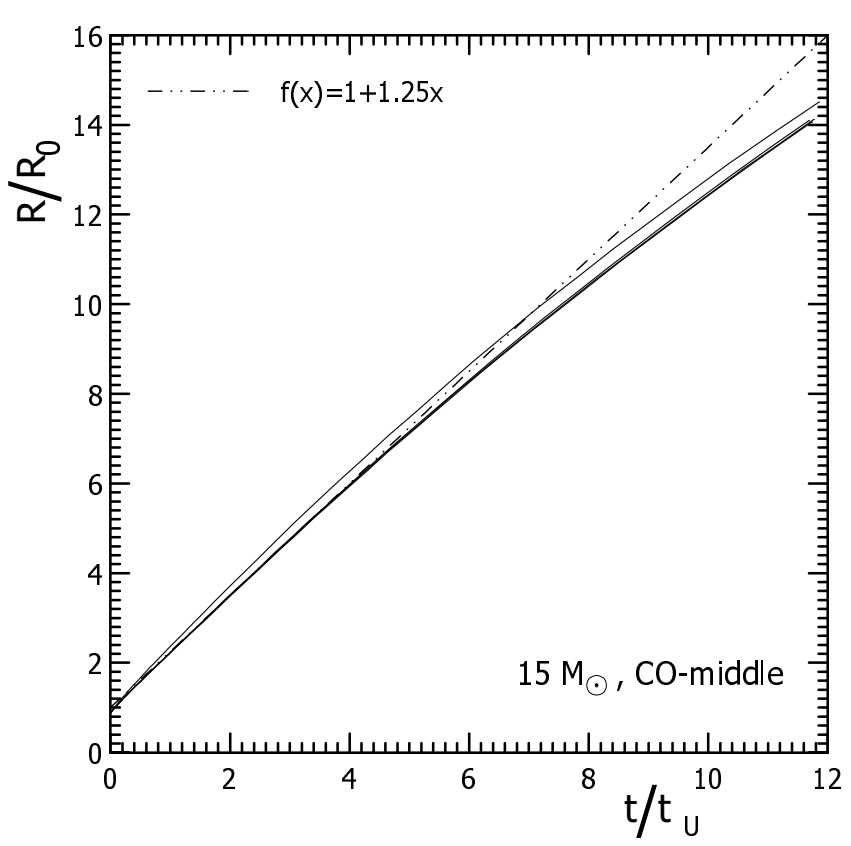

Fig. 6. Same as Fig. 2, but for the middle of the CO-shell.

lines demonstrate the approximations given by Eqs. (4) and (6) for the temperature and radius, respectively.

Inspection of Figs. 1-22 reveals a good agreement between the approximations and the hydrodynamic calculations. While finding the best values for the structural coefficients, we have focused our attention on the time interval $0 \leq t / t_{\mathrm{u}} \lesssim 2$ because the later times $\left(t / t_{\mathrm{u}} \gtrsim 2\right)$ prove to be of minor (if any) importance for nucleosynthesis since the temperature decreases by a factor of $4-5$ as compared to its peak value. From this point of view, the temperature approximation, for instance, in Fig. 7 is nearly as good as that in Fig. 5. There is, however, a certain doubt about the quality of the approximation in the 


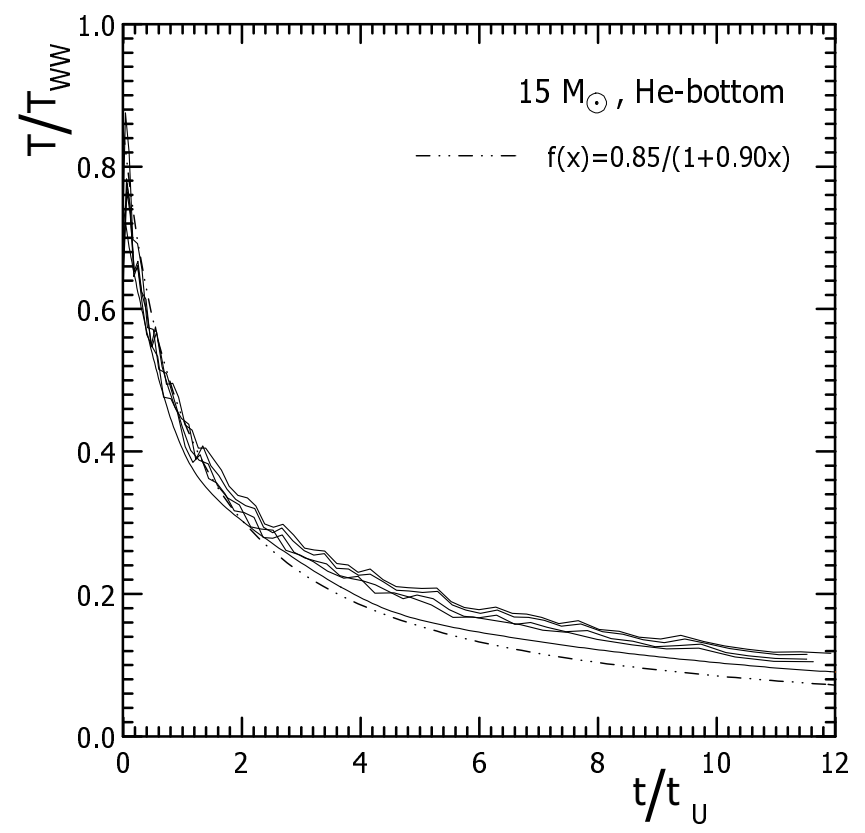

Fig. 7. Same as Fig. 5, but for the bottom of the He-shell.

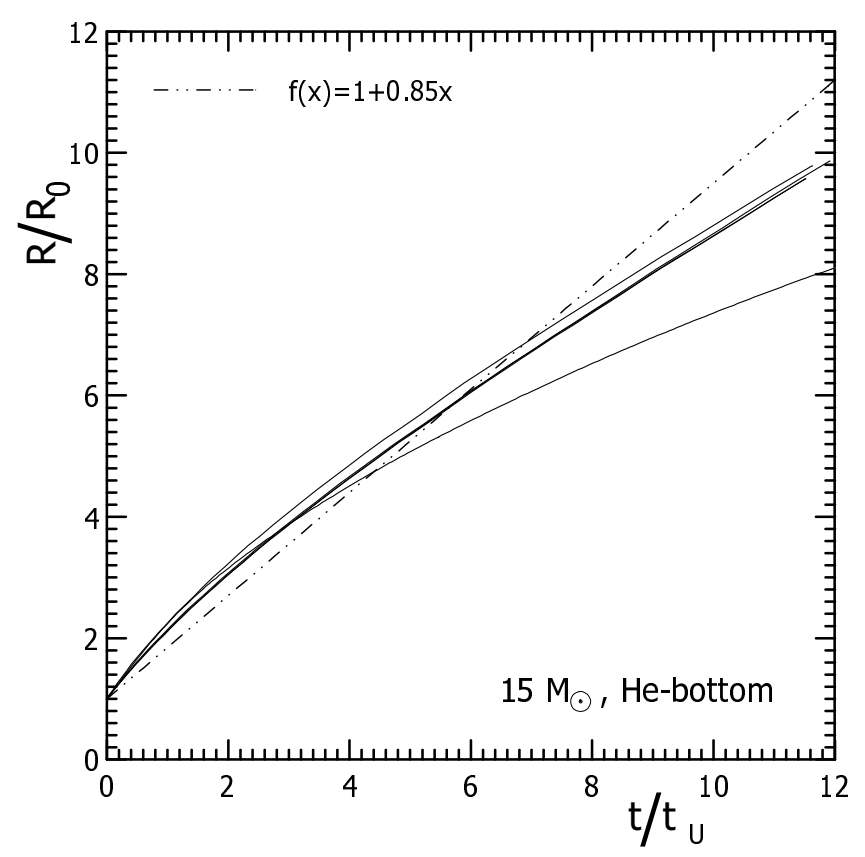

Fig. 8. Same as Fig. 6, but for the bottom of the He-shell.

middle and the top of the SiS-shell - compare Figs. 1-4 for a $15 M_{\odot}$ model and Figs. $13-16$ for a $30 M_{\odot}$ model (we remind that the top of the SiS-shell coincides with the bottom of CO-shell for a $15 M_{\odot}$ model and with that of the O-shell for a $30 M_{\odot}$ model). Although Eqs. (4) and (6) could still be useful as the first guess for the SiS-shell, the following approximate formulae for $T(t)$ and $R(t)$ turn out to be much more accurate:

$$
\begin{aligned}
& T(t)=\frac{T_{\mathrm{p}}}{\sqrt{1+\xi_{\mathrm{T} 1} t / t_{\mathrm{u}}}}, \quad T_{\mathrm{p}}=\xi_{\mathrm{p}} T_{\mathrm{WW}}, \\
& R(t)=R_{0}\left(\xi_{\mathrm{r} 1} t / t_{\mathrm{u}}+\sqrt{1+\xi_{\mathrm{r} 2} t / t_{\mathrm{u}}}\right) .
\end{aligned}
$$

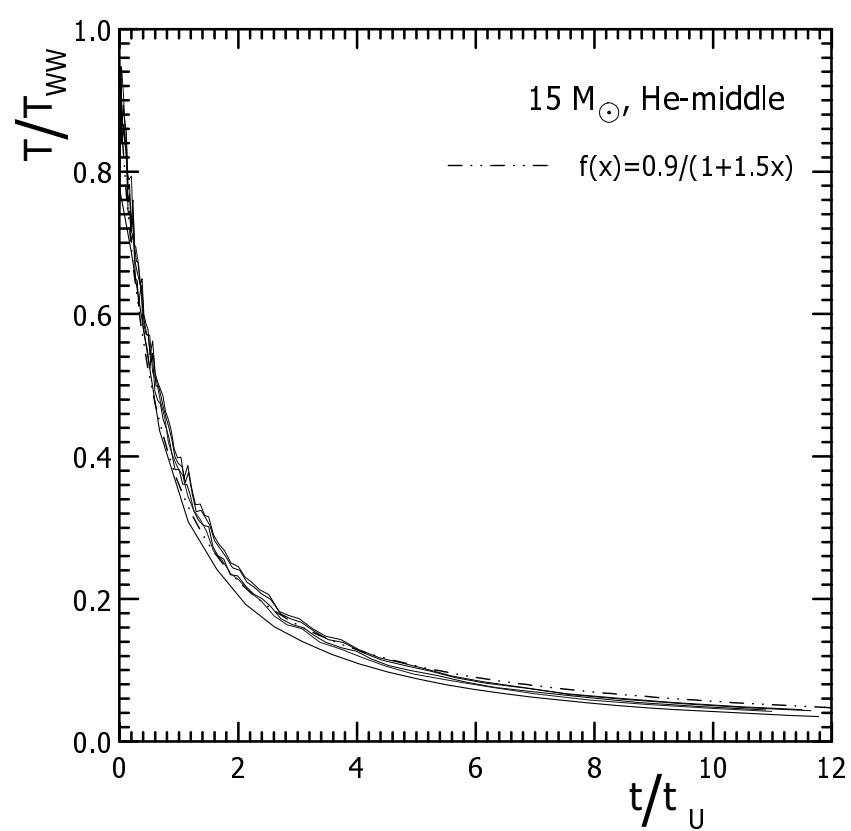

Fig. 9. Same as Fig. 5, but for the middle of the He-shell.

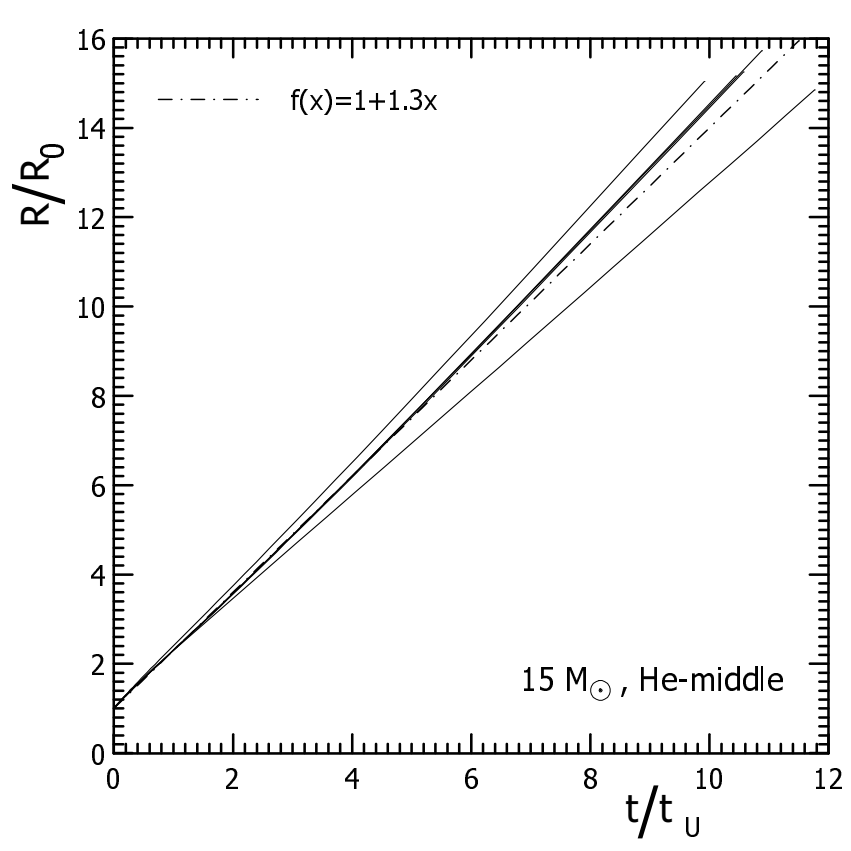

Fig. 10. Same as Fig. 6, but for the middle of the He-shell.

The structural coefficients $\xi_{\mathrm{T} 1}, \xi_{\mathrm{r} 1}$, and $\xi_{\mathrm{r} 2}$ are given in parenthesis in Tables 3 and 4 . These approximations are shown in Figs. 1-4, 13-16 by black dots (not connected by lines!). One can see an excellent agreement for the temperature curves and a fairly good one for the radius curves.

Figures 23 and 24 demonstrate that the exponential approximation based on the "hydrodynamic" time scale $\tau$ (Eq. (1)) is not actually adequate. It predicts a much slower decrease in the post-shock temperature, even when one uses the peak density $\rho_{\mathrm{p}}=7 \rho_{0}$ to evaluate $\tau$ (the curves labelled by number 2 in Figs. 23 and 24) rather than the initial pre-shock density $\rho_{0}$ (the curves labelled by 1 ). 


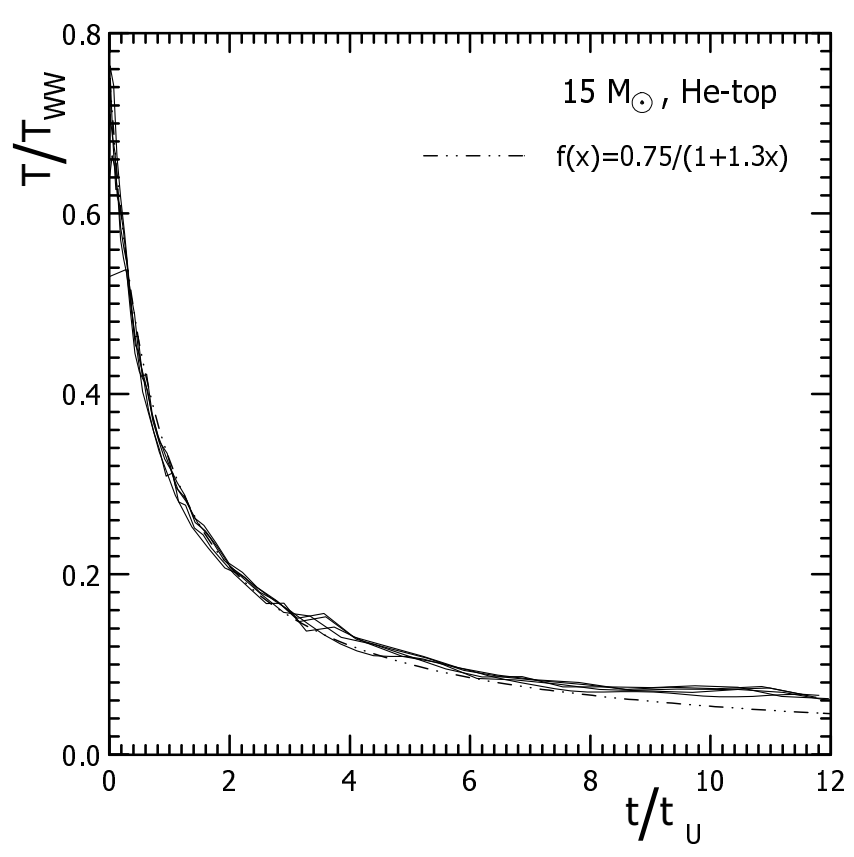

Fig. 11. Same as Fig. 5, but for the top of the He-shell.

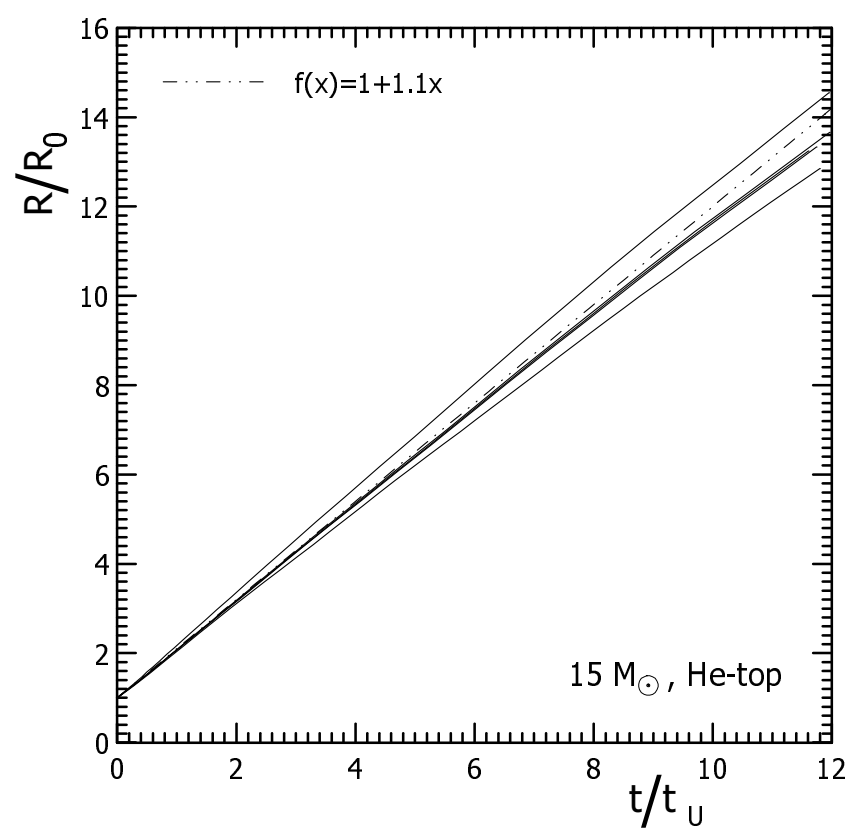

Fig. 12. Same as Fig. 6, but for the top of the He-shell.

The discrepancy is especially pronounced for the helium shell (Fig. 24). The curves 1 and 2 in Figs. 23 and 24 are drawn for a standard explosion energy $E=1.5 \times 10^{51} \mathrm{erg}$.

The density approximation given by Eq. (5) remains to be discussed. Since the density is not as important for nucleosynthesis as the temperature, the approximation should not be necessarily of high accuracy. From this point of view Eq. (5) can serve as an appropriate approximation.

In Table 5, one can find the numerical values of the parameters involved in our consideration for the case of a standard supernova explosion energy of $E=1.5 \times 10^{51} \mathrm{erg}$. The SW compression $\rho_{\mathrm{p}} / \rho_{0}$ given in the last column of

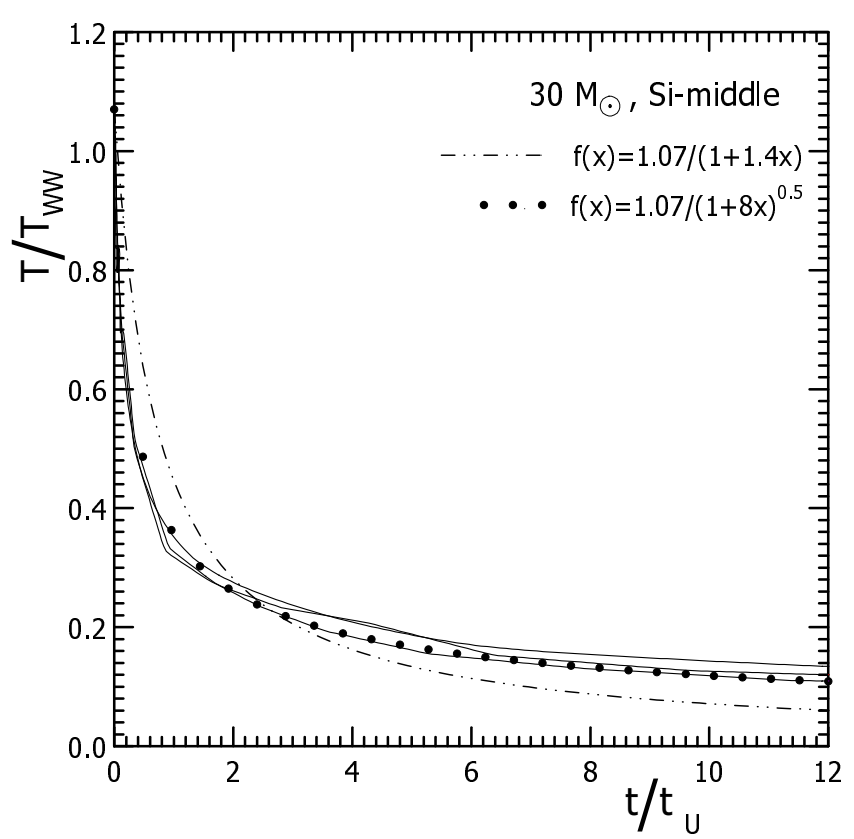

Fig. 13. Same as Fig. 1, but for a $30 M_{\odot}$ model.

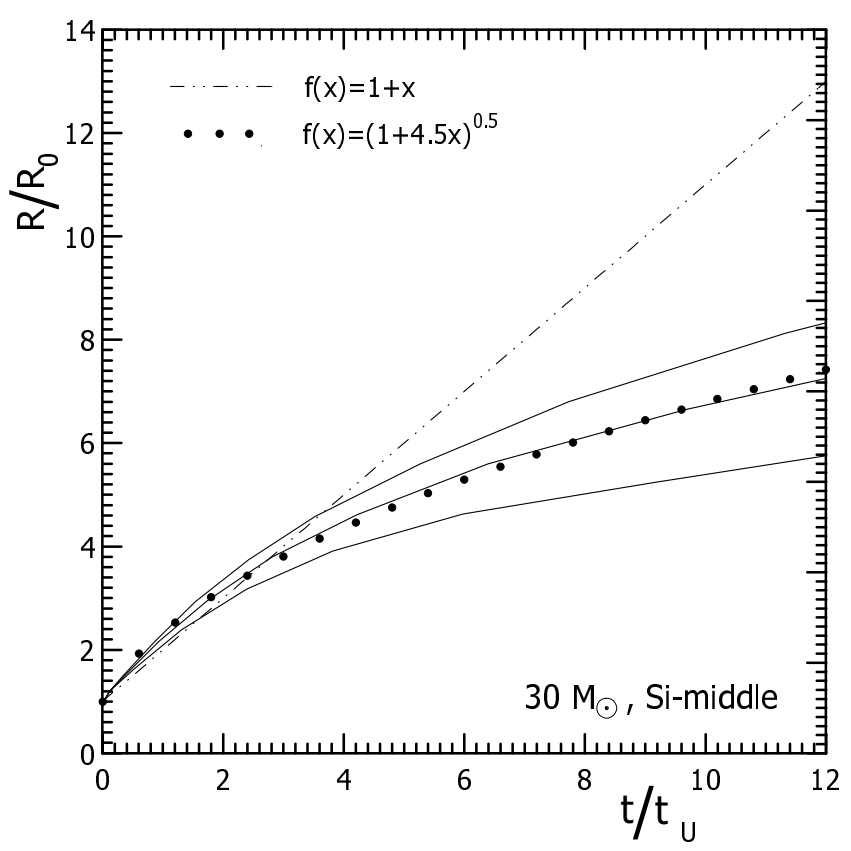

Fig. 14. Same as Fig. 2, but for a $30 M_{\odot}$ model.

Table 5 was calculated from the Hugoniot conditions using corresponding values of $T_{\mathrm{p}}$ (the third column) and $\rho_{0}$ (from Tables 1 and 2). One can see that the approximation $\rho_{\mathrm{p}} / \rho_{0}=7$ is not far from reality. Still we have to verify how the assumption $\rho(t) \sim T^{3}(t)$ actually works. Figure 25 shows the divergence $F_{\rho}$ of actual $\rho(t)$ from the approximation given by Eq. (5):

$F_{\rho}=\frac{\rho(t)}{\rho_{\mathrm{p}}}\left(\frac{T_{\mathrm{p}}}{T}\right)^{3}$,

where the "isentropic" density $\rho(t)$ is calculated from the condition that entropy remains constant in every 


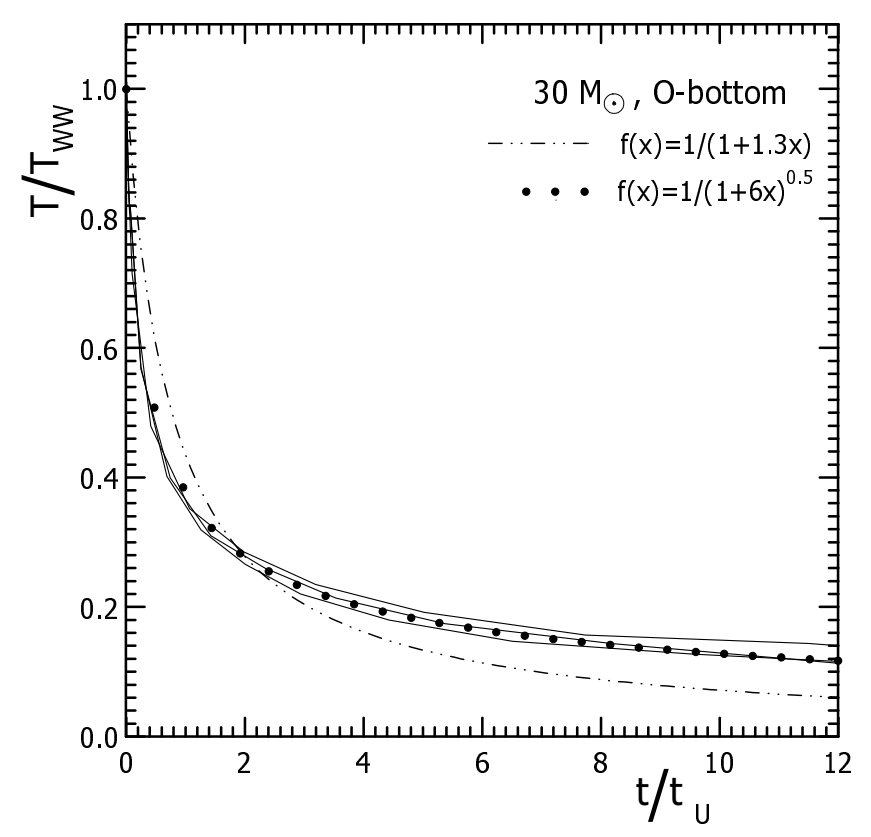

Fig. 15. Same as Fig. 3, but for a $30 M_{\odot}$ model.

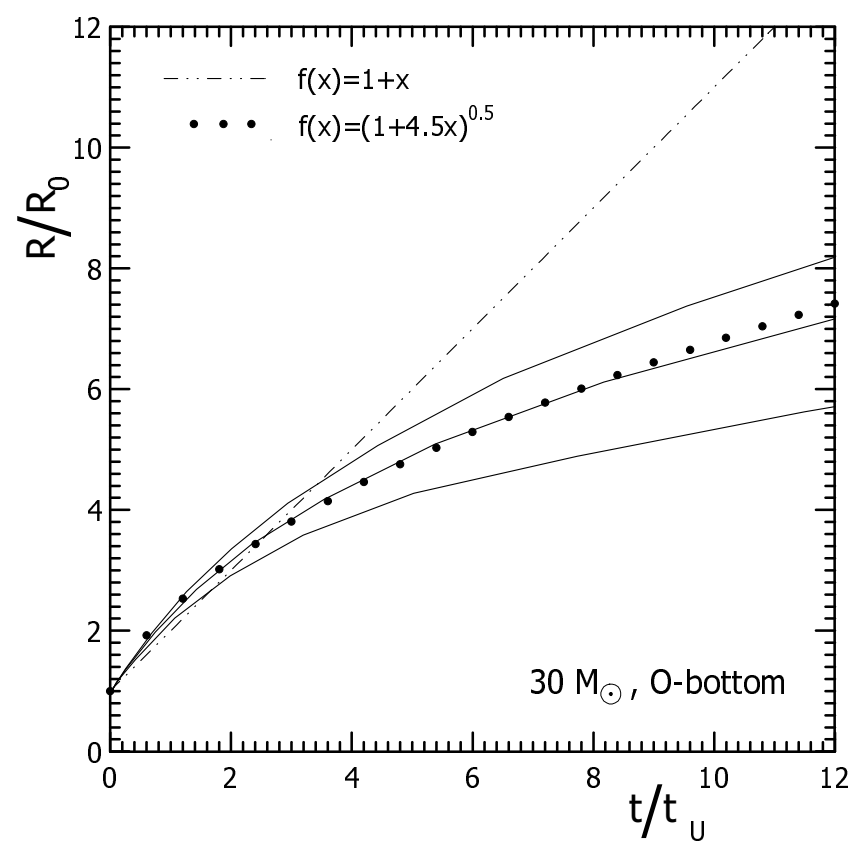

Fig. 16. Same as Fig. 4, but for a $30 M_{\odot}$ model.

Lagrangian layer during its post-shock expansion: $S[\rho, T(t)]=S\left(\rho_{\mathrm{p}}, T_{\mathrm{p}}\right)=S_{\mathrm{p}}=$ const. Here $S_{\mathrm{p}}$ is the SW peak entropy and $T=T(t)$ is defined either by Eq. (4) or (8). If $\rho(t)$ followed strictly the law $\rho \sim T^{3}, F_{\rho}$ would remain all the time equal to 1 . We see that for a $15 M_{\odot}$ model the deviation from this law is virtually insignificant - it remains in the limits $\pm 30 \%$. However in the Heand O-shell of a $30 M_{\odot}$ model the "isentropic" density is decreasing more slowly than $T^{3}$. Nevertheless, the approximation $\rho \sim T^{3}$ still is acceptable even in the O-shell of a $30 M_{\odot}$ model if one remembers that nucleosynthesis occurs efficiently in the time interval $0 \leq t / t_{\mathrm{u}} \lesssim 2-$ at $t / t_{\mathrm{u}}=2$ the post-shock temperature and density have fallen typically by a factor of $\sim 5$ and $\sim 100$, respectively.

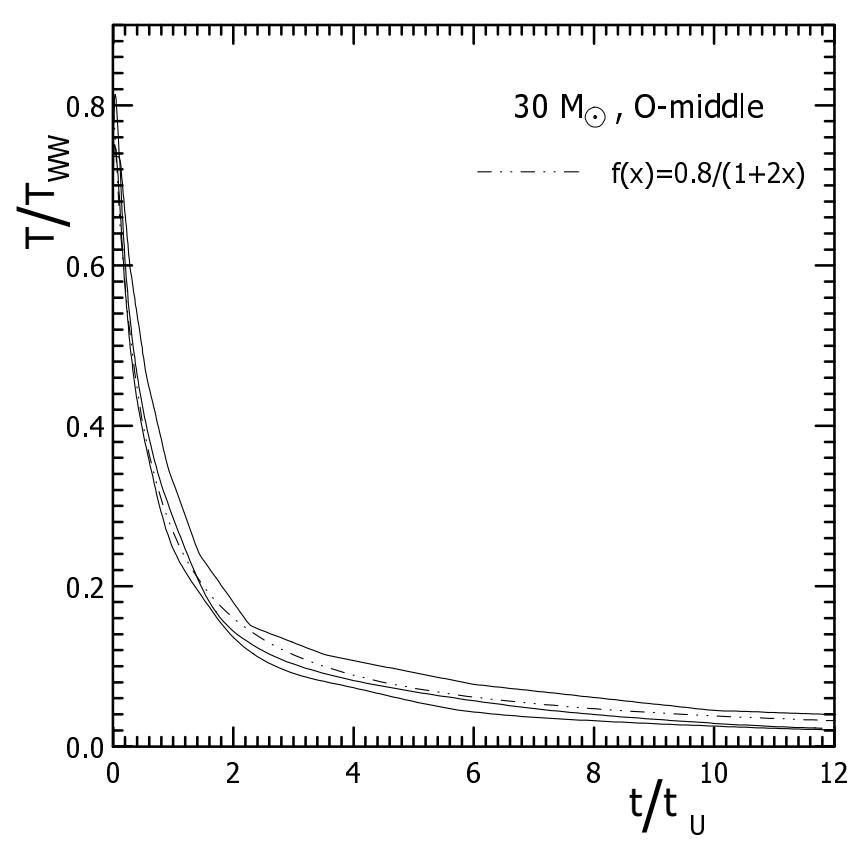

Fig. 17. Same as Fig. 5, but for a $30 M_{\odot}$ model.

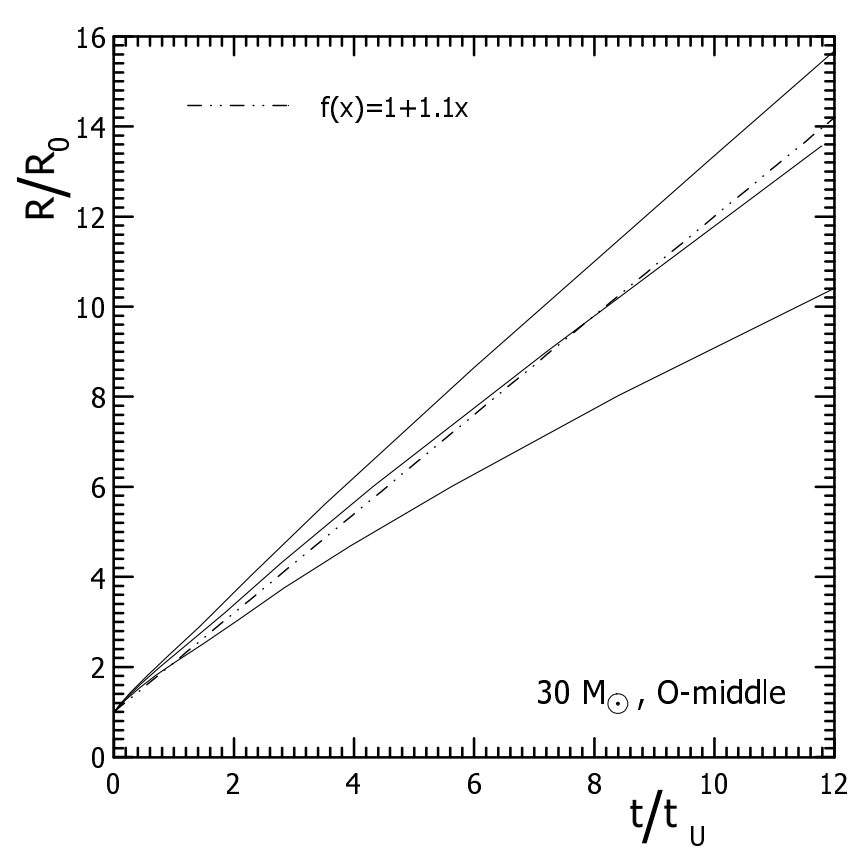

Fig. 18. Same as Fig. 6, but for a $30 M_{\odot}$ model.

Physically, the density-temperature relation at constant entropy $\left(\rho \sim T^{\frac{1}{\Gamma-1}}\right)$ is determined by the adiabatic index $\Gamma$ that is defined as

$\Gamma=1+\left(\frac{\partial \log T}{\partial \log \rho}\right)_{S}=1+\gamma \nabla_{\mathrm{A}}$,

with $\gamma \equiv\left(\frac{\partial \log P}{\partial \log \rho}\right)_{S}, \quad \nabla_{\mathrm{A}} \equiv\left(\frac{\partial \log T}{\partial \log P}\right)_{S}$.

Figure 26 shows $\Gamma$ along isentropes $S=S_{\mathrm{p}}$. We see that $\Gamma$ is not very different from $4 / 3$ and, therefore, the approximation $\rho \sim T^{3}$ turns out to be not so far from reality. The exception is the O-shell of a $30 M_{\odot}$ model where $\rho \sim T^{2}$ 


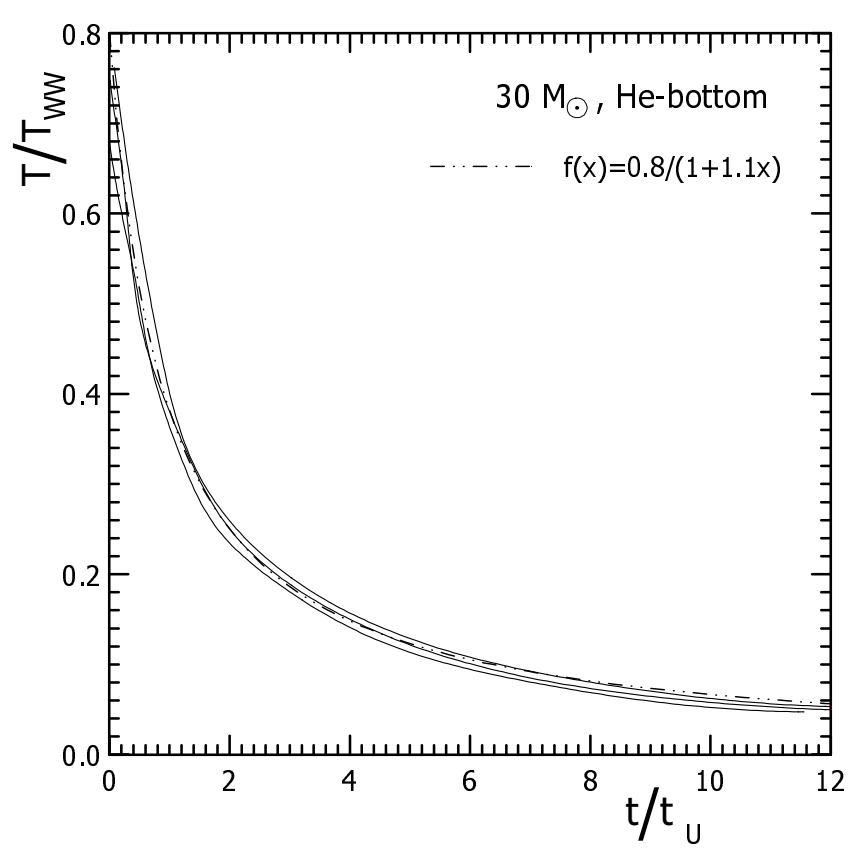

Fig. 19. Same as Fig. 7, but for a $30 M_{\odot}$ model.

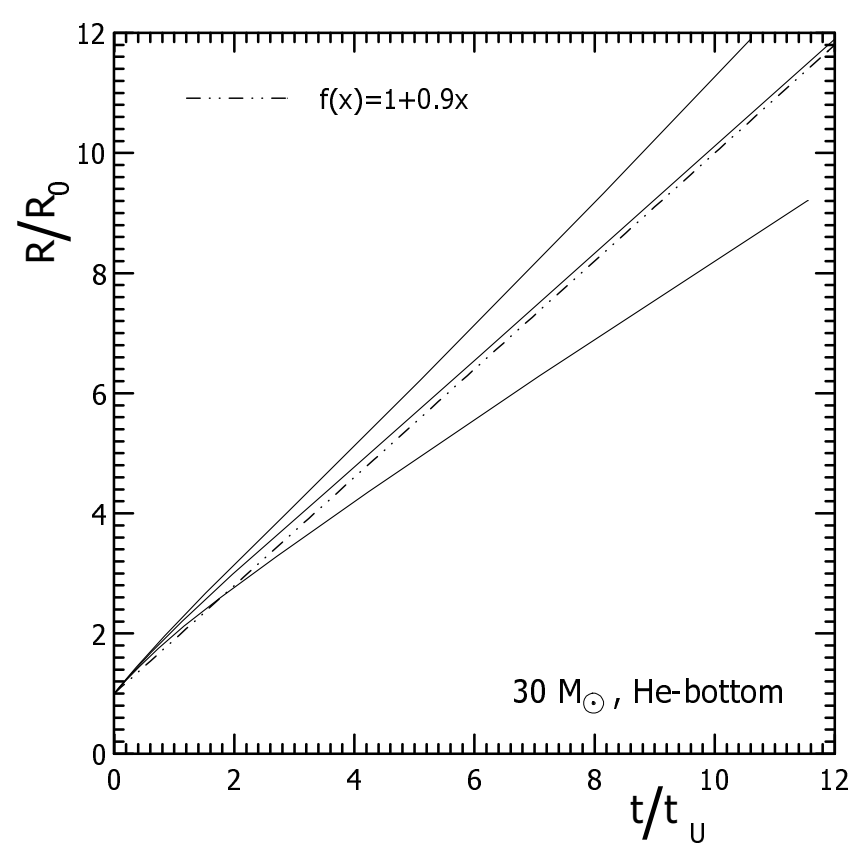

Fig. 20. Same as Fig. 8, but for a $30 M_{\odot}$ model.

proves to be a more appropriate approximation since there $\Gamma$ is close to 1.5 rather than 1.33 . Note that at early postshock times $t / t_{\mathrm{u}} \lesssim 4, \Gamma$ becomes less than $4 / 3$ in the SiSand CO-shell. This happens owing to the contribution of the electron-positron pairs into the equation of state. For example in the case of a $15 M_{\odot}$ model the number of pairs per "atomic" electron is equal to about 0.7 (SiS-middle) and 0.6 (CO-middle) for the peak values of $T_{\mathrm{p}}$ (Table 5) and $\rho_{\mathrm{p}}$ (Tables 1,5$)$. Thus, the total number of leptons (electrons plus positrons) increases by a factor of $\approx 2$.

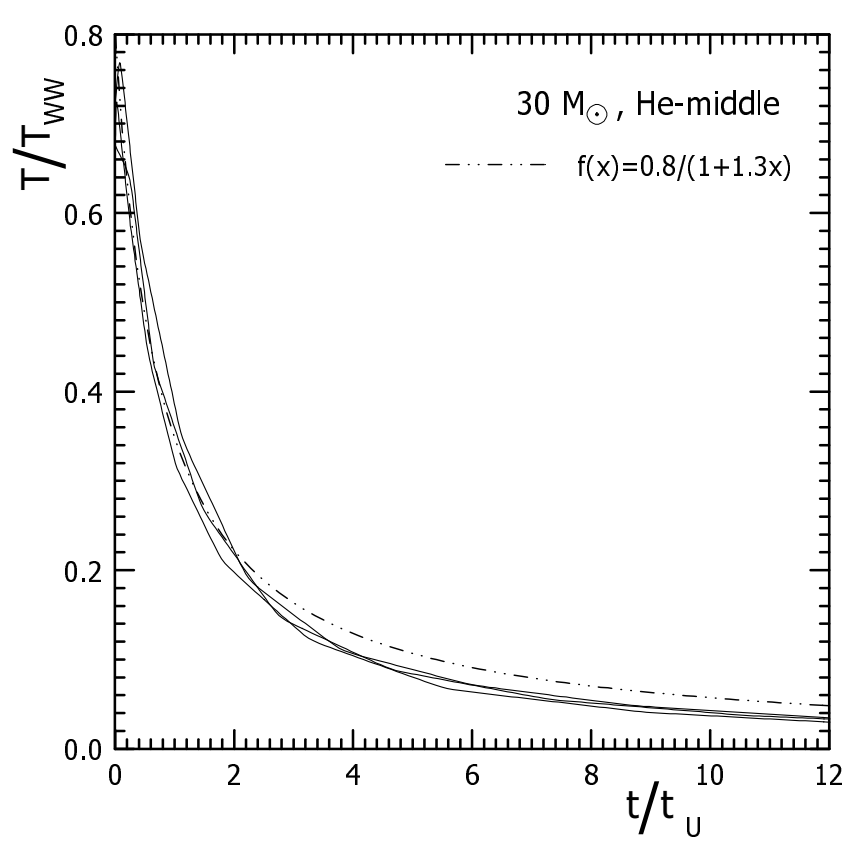

Fig. 21. Same as Fig. 9, but for a $30 M_{\odot}$ model.

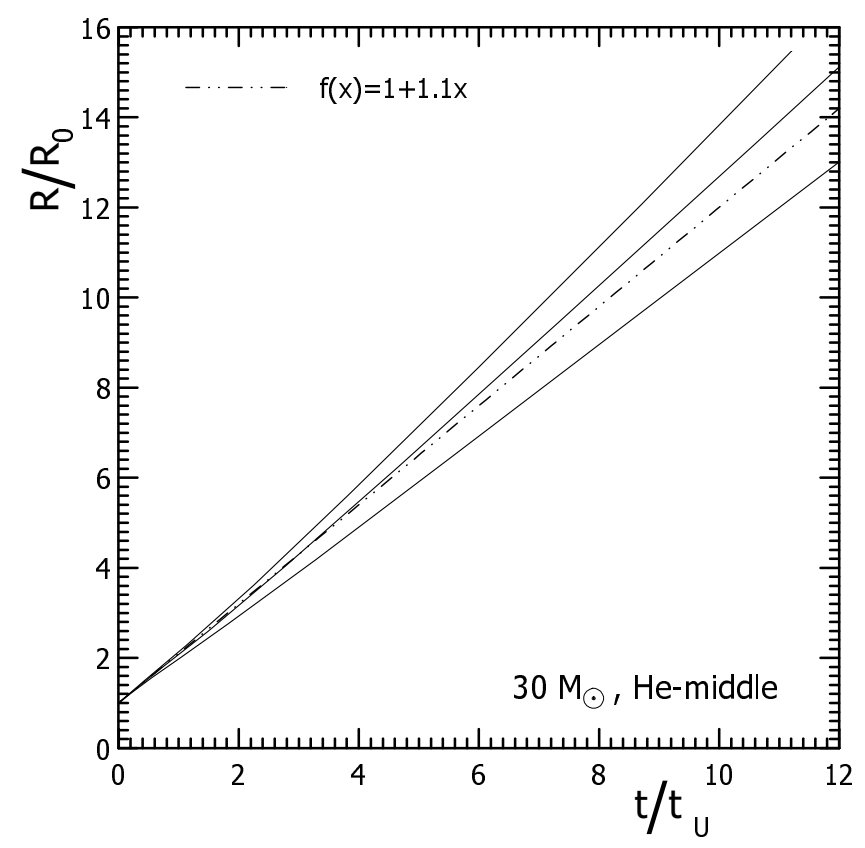

Fig. 22. Same as Fig. 10, but for a $30 M_{\odot}$ model.

\section{Conclusions}

The approximation formulae presented here are believed to be useful for the astrophysicists and nuclear physicists working in the field of stellar nucleosynthesis. In order to adjust their nuclear yields to the supernova models, they have to specify only two basic parameters: radius $R_{0}$ and density $\rho_{0}$ for a presupernova layer of interest, and to choose the supernova explosion energy $E$. Then with the Weaver-Woosley temperature $T_{\mathrm{WW}}$ (Eq. (2)) and characteristic time scale $t_{\mathrm{u}}$ (Eq. (3)) known, the temporal behavior of temperature, density, and radius of the 


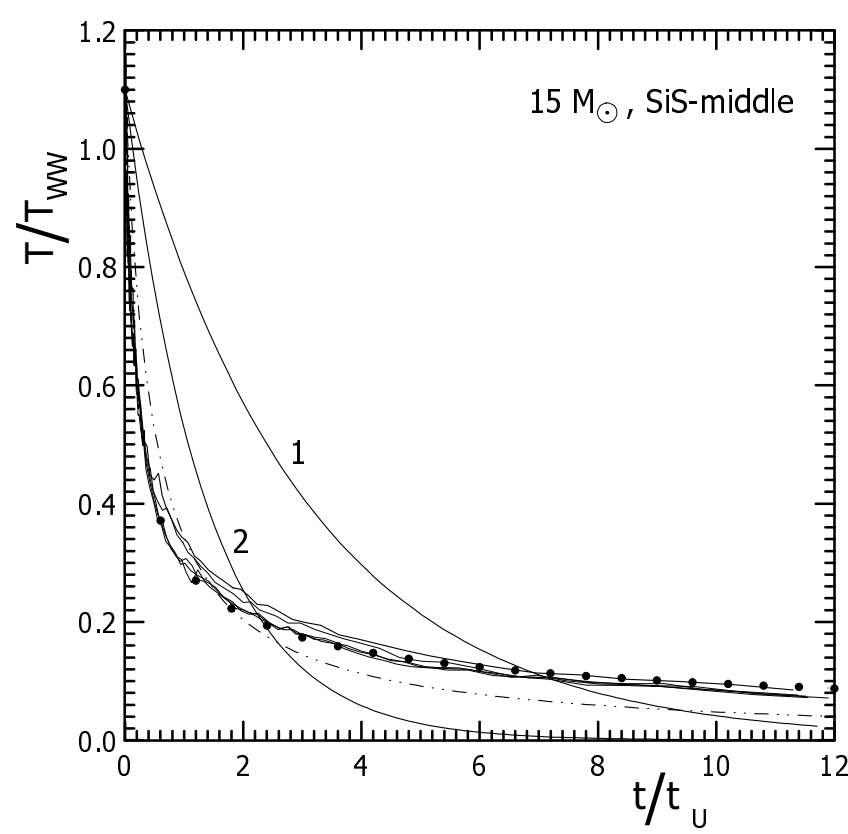

Fig. 23. The comparison of the post-shock temperature in the middle of SiS-shell from Fig. 1 with a simplified "hydrodynamic" approximation shown by curves 1 and 2 (see text).

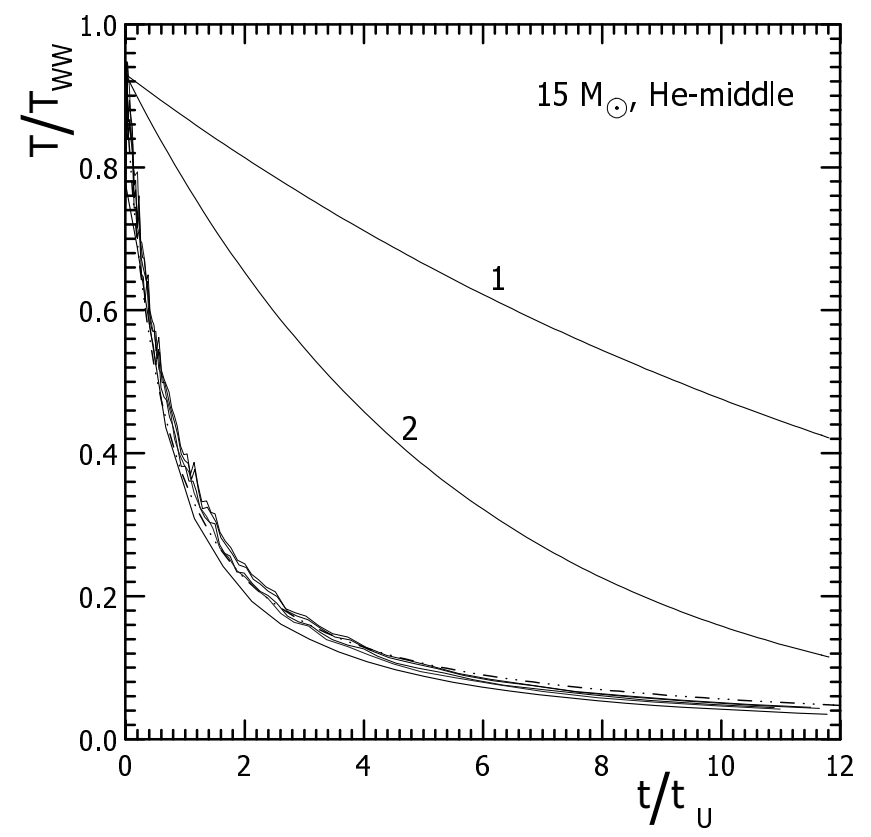

Fig. 24. Same as Fig. 23, but in the middle of the He-shell from Fig. 9.

Lagrangian layer in question proves to be determined unambiguously by Eqs. (4)-(6), (8), (9). The corresponding structural coefficients are given in Tables 3 and 4 for a $15 M_{\odot}$ and $30 M_{\odot}$ supernova, respectively. Although these coefficients are dependent on the supernova mass, the dependence is not so crucial. In the first rough approxima-

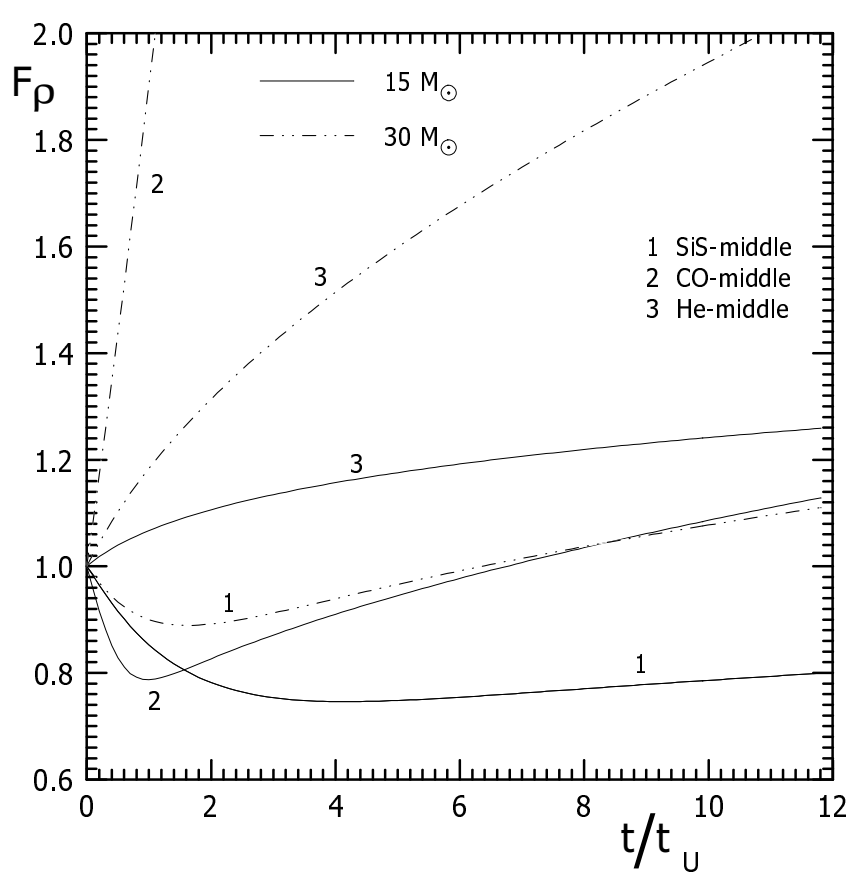

Fig. 25. The divergence of the post-shock density from the approximation given by Eq. (5).

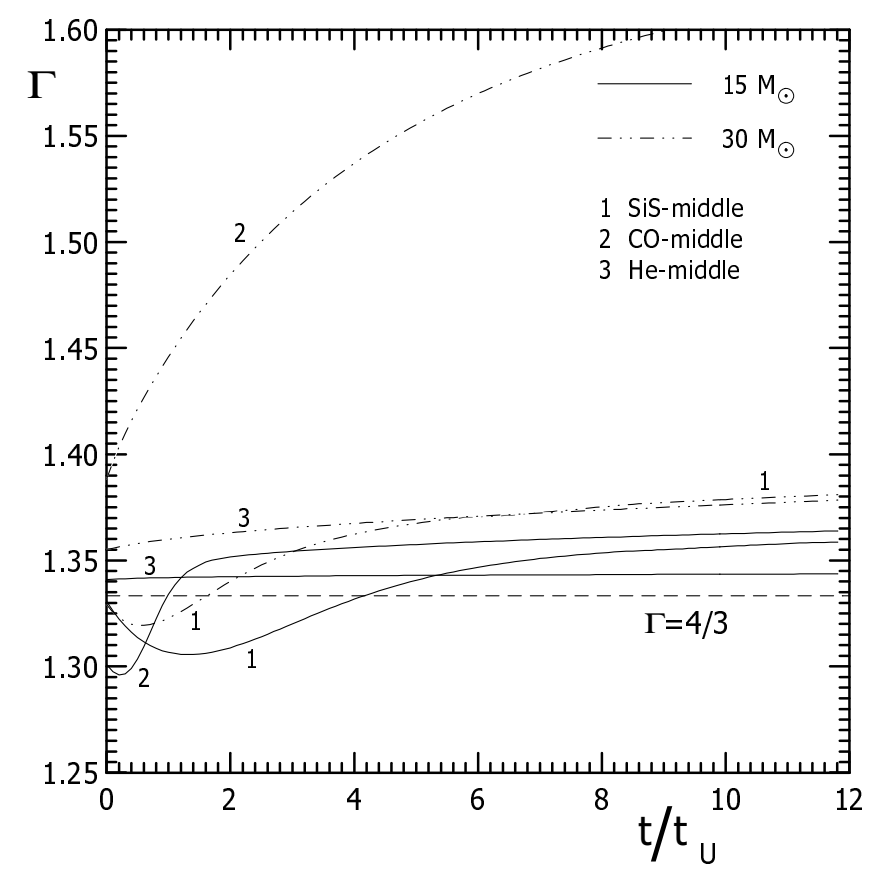

Fig. 26. Adiabatic index $\Gamma$ versus time for the post-shock expansion. The level $\Gamma=4 / 3$ is shown by a horizontal dashed line.

tion, one may neglect this dependence assuming the coefficients for a $15 M_{\odot}$ model as standard values. In the future, it would be worth-while to investigate in detail the dependence of the structural coefficients on presupernova mass, metallicity, and stellar rotation. 
Table 5. The shock wave parameters for $15 M_{\odot}$ and $30 M_{\odot}$ supernovae with the explosion energy $1.5 \times 10^{51} \mathrm{erg}$.

\begin{tabular}{lcccccc}
\hline \hline \multicolumn{1}{c}{ Shell } & $\begin{array}{c}M \\
\left(M_{\odot}\right)\end{array}$ & $\begin{array}{c}T_{\mathrm{p}} \\
(\mathrm{K})\end{array}$ & $\begin{array}{c}T_{\mathrm{WW}} \\
(\mathrm{K})\end{array}$ & $\begin{array}{c}t_{\mathrm{u}} \\
(\mathrm{s})\end{array}$ & $\begin{array}{c}\Delta t_{\mathrm{SW}} \\
(\mathrm{s})\end{array}$ & $\begin{array}{c}\rho_{\mathrm{p}} \\
\left(\rho_{0}\right)\end{array}$ \\
\hline SiS-middle & 15 & $1.00 \times 10^{10}$ & $9.10 \times 10^{9}$ & 0.158 & 0.0453 & 6.7 \\
SiS-middle & 30 & $6.63 \times 10^{9}$ & $6.20 \times 10^{9}$ & 0.374 & 0.0620 & 6.4 \\
\hline SiS-top & 15 & $8.65 \times 10^{9}$ & $7.86 \times 10^{9}$ & 0.184 & 0.0815 & 6.7 \\
SiS-top & 30 & $5.24 \times 10^{9}$ & $5.24 \times 10^{9}$ & 0.482 & 0.115 & 6.2 \\
\hline CO-middle & 15 & $2.76 \times 10^{9}$ & $2.90 \times 10^{9}$ & 0.797 & 0.778 & 6.7 \\
O-middle & 30 & $9.70 \times 10^{8}$ & $1.21 \times 10^{9}$ & 6.79 & 5.53 & 5.1 \\
\hline CO-top & 15 & $8.84 \times 10^{8}$ & $1.04 \times 10^{9}$ & 2.21 & 4.72 & 6.5 \\
O-top & 30 & $5.00 \times 10^{8}$ & $6.24 \times 10^{8}$ & 10.2 & 17.5 & 5.7 \\
\hline He-middle & 15 & $2.93 \times 10^{8}$ & $3.26 \times 10^{8}$ & 18.3 & 28.9 & 6.5 \\
He-middle & 30 & $2.90 \times 10^{8}$ & $3.62 \times 10^{8}$ & 23.2 & 40.7 & 5.9 \\
\hline He-top & 15 & $9.75 \times 10^{7}$ & $1.30 \times 10^{8}$ & 32.2 & 126 & 6.9 \\
He-top & 30 & $1.04 \times 10^{8}$ & $1.30 \times 10^{8}$ & 19.2 & 133 & 7.0 \\
\hline
\end{tabular}

Acknowledgements. It is a pleasure to express our deep gratitude to the Max-Planck-Institut für Astrophysik for hospitality and financial support. The work was supported also by the Swiss National Science Foundation and the Russian Foundation for Basic Research (Project 00-02-17230). We are grateful to G. A. Tammann and the referee for useful suggestions.

\section{References}

Blinnikov, S. I., Dunina-Barkovskaya, N. G., \& Nadyozhin, D. K. 1996, ApJS, 106, 171

Deputovich, A. Yu., \& Nadyozhin, D. K. 1999, Pis'ma Astr. Zh., 25, 750 [Astron. Lett., 25, 649]

Litvinova, I. Yu., \& Nadyozhin, D. K. 1983, Ap\&SS, 89, 89

Nadyozhin, D. K., Panov, I. V., \& Deputovich, A. Yu. 2000, in Proceedings of the 10th Workshop on Nuclear Astrophysics, Ringberg Castle, Tegernsee, Germany, ed. W. Hillebrandt, \& E. Müller, MPA/P12, 99

Nadyozhin, D. K., Panov, I. V., \& Blinnikov, S. I. 1998, A\&A, 335,207

Thielemann, F.-K., Arnold, M., \& Hillebrandt, W. 1979, A\&A, 74,175

Wallerstein, G., Iben, I. Jr, Parker, P., et al. 1997, Rev. Mod. Phys., 69, 995

Weaver, T. A., \& Woosley, S. E. 1980, Ann. NY Acad. Sci., 336,335

Woosley, S. E., \& Weaver, T. A. 1995, ApJS, 101, 181 